\title{
Visuospatial memory impairment as a potential neurocognitive marker to predict tau pathology in Alzheimer's continuum
}

\author{
Eun Hyun Seo ${ }^{1,2 \dagger}$, Ho Jae Lim ${ }^{1,3 \dagger}$, Hyung-Jun Yoon ${ }^{4 \dagger}$, Kyu Yeong Choi ${ }^{1}$, Jang Jae Lee ${ }^{1}$, Jun Young Park ${ }^{5,11}$, \\ Seong Hye Choi ${ }^{6}$, Hoowon Kim ${ }^{7}$, Byeong C. Kim ${ }^{8}$ and Kun Ho Lee ${ }^{1,9,10,11^{*}}$ (D)
}

\begin{abstract}
Background: Given that tau accumulation, not amyloid- $\beta(A \beta)$ burden, is more closely connected with cognitive impairment in Alzheimer's disease (AD), a detailed understanding of the tau-related characteristics of cognitive function is critical in both clinical and research settings. We investigated the association between phosphorylated tau ( $p$-Tau) level and cognitive impairment across the AD continuum and the mediating role of medial temporal lobe (MTL) atrophy. We also developed a prediction model for abnormal tau accumulation.
\end{abstract}

Methods: We included participants from the Gwangju Alzheimer's Disease and Related Dementia Cohort in Korea, who completed cerebrospinal fluid analysis and clinical evaluation, and corresponded to one of three groups according to the biomarkers of A and T profiles based on the National Institute on Aging and Alzheimer's Association research framework. Multiple linear and logistic regression analyses were performed to examine the association between p-Tau and cognition and to develop prediction models. Receiver operating characteristic curve analysis was performed to examine the discrimination ability of the models.

Results: Among 185 participants, 93 were classified as A-T-, 23 as A+T-, and 69 as A+T+. There was an association between decreased visuospatial delayed memory performance and $p$-Tau level $(B=-0.754, \beta=-0.363, p<0.001)$, independent of other relevant variables (e.g., $A \beta$ ). MTL neurodegeneration was found to mediate the association between the two. Prediction models with visuospatial delayed memory alone (area under the curve $[A \cup C]=0.872$ ) and visuospatial delayed memory and entorhinal thickness $(A U C=0.921)$ for abnormal tau accumulation were suggested and they were validated in an independent sample (AUC $=0.879$ and 0.891 , respectively).

Conclusion: It is crucial to identify sensitive cognitive measures that capture subtle cognitive impairment associated with underlying pathological changes. Preliminary findings from the current study might suggest that abnormal tau accumulation underlies episodic memory impairment, particularly visuospatial modality, in the AD continuum. Suggested models are potentially useful in predicting tau pathology, and might be utilized practically in the field.

Keywords: Alzheimer's disease, ATN classification, Cerebrospinal fluid, Tau, Visuospatial memory, Biomarkers

*Correspondence: leekho@chosun.ac.kr

†Eun Hyun Seo, Ho Jae Lim and Hyung-Jun Yoon contributed equally to this work.

${ }^{1}$ Gwangju Alzheimer's Disease and Related Dementia Cohort Research Center, Chosun University, 61452 Gwangju, Republic of Korea

Full list of author information is available at the end of the article

\section{Background}

Biomarker identification for Alzheimer's disease (AD) has evolved over the past few decades. Accordingly, the research framework for $\mathrm{AD}$ has changed dramatically [1]. It is moving from a clinically defined to a biologically defined disease that is understood to begin considerably original author(s) and the source, provide a link to the Creative Commons licence, and indicate if changes were made. The images or other third party material in this article are included in the article's Creative Commons licence, unless indicated otherwise in a credit line to the material. If material is not included in the article's Creative Commons licence and your intended use is not permitted by statutory regulation or exceeds the permitted use, you will need to obtain permission directly from the copyright holder. To view a copy of this licence, visit http://creativecommons.org/licenses/by/4.0/. The Creative Commons Public Domain Dedication waiver (http://creativeco mmons.org/publicdomain/zero/1.0/) applies to the data made available in this article, unless otherwise stated in a credit line to the data. 
earlier than the onset of cognitive decline. This framework enables us to define the earliest stages of the disease continuum. In addition, with a biomarker-based definition of $\mathrm{AD}$, the mechanisms underlying the clinical manifestations of $\mathrm{AD}$ can be understood more precisely. Clinically observed cognitive symptoms are not always specific to AD. However, the characteristics of cognitive impairment that are closely associated with AD neuropathological processes can be explored.

Amyloid-beta $(A \beta)$ plaques and tau neurofibrillary tangles (NFT) are known to be the hallmarks of AD pathology, and they begin to accumulate in the brain years before clinical symptoms [2]. A $\beta$ deposition, pathologic tau, and neurodegeneration have been served as biomarkers to define $\mathrm{AD}$, which lead $\mathrm{AT}(\mathrm{N})$ classification system [1]. Moreover, many studies using cerebrospinal fluid (CSF) demonstrated such classification or combination of the three biomarkers have prognostic utility as well as diagnostic value [3-5]. In addition, ratio between $A \beta_{1-42}$ and phosphorylated tau (p-Tau) also showed association with subsequent cognitive decline in cognitively normal (CN) or mild cognitive impairment (MCI) older adults [3, 5]. Over the past decade, accumulating evidence has suggested that early cognitive changes are more closely associated with tau pathology than $\mathrm{A} \beta$ pathology. Transgenic mouse model [6], clinicopathological [7], and neuroimaging [8-10] studies support that $A \beta$ is the initial factor, and clinical symptoms begin to appear as tau pathology progresses. Especially, a number of studies have reported that tau accumulation could be a highly predictive indicator for future cognitive decline in both nondemented $[8,11]$ and $\mathrm{AD}[12]$. Given that tau accumulation, not $\mathrm{A} \beta$ burden, is more closely connected with cognitive change in the AD continuum [13-15], a detailed understanding of the relationship between tau accumulation and specific cognitive function is critical for effectively capturing AD-related symptoms at the earliest stage of the disease.

Several recent studies have attempted to determine whether there are tau-related cognitive impairments, but their findings have been inconclusive. Many studies have reported associations between tau pathology and episodic memory $[8,11,16,17]$. For example, two studies using CSF p-Tau demonstrated significant associations between episodic memory and tau levels $[17,18]$. Studies using tau positron emission tomography (PET) also reported that elevated tau deposition in the medial temporal lobe (MTL) was associated with episodic memory impairment $[8,9]$. However, other studies have shown a significant association between tau pathology and executive function, not episodic memory [19], or no association with any of the cognitive domain [20,21]. The specific tau-associated cognitive symptoms in individuals with $\mathrm{AD}$ continuum remain unclear.
Although several attempts have been made to shed light on tau-associated cognitive impairment in aging populations, previous studies have had several limitations. Some studies have used relatively brief cognitive tests $[18,21]$ or only memory indexes [22]. Visuospatial memory tests were not included in most previous studies [8-10, 16, 18-22]. More comprehensive neuropsychological measures that assess multiple cognitive domains should be utilized. In addition, in some studies, tau-associated cognitive impairment was investigated without controlling for the influence of $A \beta$ pathology $[9,16]$. Due to concomitant tau and $A \beta$ pathology, the influence of $A \beta$ pathology on cognitive function should be controlled to investigate tau-specific cognitive impairment.

Given that NFT initially forms in MTL regions [23, 24] and that tau pathology is closely linked to longitudinal cortical thinning, especially in MTL [25], atrophy in such regions could play a role in cognitive impairment. One recent investigation reported both direct and indirect (i.e., gray matter-mediated) tau effects on cognition [26]. Although tau-associated cognitive impairment could be mediated by gray matter loss in such brain structures, only a few studies have investigated this mediated association.

Tau accumulation is generally measured by either tau PET or CSF p-Tau levels. However, these measurements are not always feasible, especially in community-based settings. Noninvasive and cost-effective markers with good predictability for abnormal tau accumulation, i.e., biomarker " $T$ " positive, are highly desirable. However, to date, few studies have explored prediction models for abnormal tau accumulation based on clinical information that is relatively easy to obtain.

Therefore, we aimed to investigate the specific association between p-Tau level and cognitive function across the $\mathrm{AD}$ continuum and to examine the mediating effects of MTL neurodegeneration on this association. We also developed prediction models for abnormal tau accumulation to facilitate the use of specific neuropsychological tests in the field. Finally, the validities of the prediction models were tested on an independent dataset.

\section{Methods \\ Participants}

This is a cross-sectional study. We recruited individuals who had agreed to undergo lumbar puncture in a pool of older adults registered at the Gwangju Alzheimer's Disease and Related Dementia (GARD) Cohort from December 2014 to October 2019. The GARD database has been described previously [27, 28]. Briefly, inclusion criteria in GARD were as follows: for $\mathrm{CN}$ participants (1) aged 60 and more, (2) a clinical dementia rating (CDR) score of $0,(3)$ a normal range of cognitive 
function, i.e., all neuropsychological tests z-scores were above -1.5 standard deviation (SD) according to age-, education-, and gender-specific norms; for MCI (1) aged 60 and more, (2) a CDR score of 0.5, (3) meet MCI criteria by Winblad [29]; and for AD dementia (1) a CDR score of 0.5 and more, (2) meet the Diagnostic and Statistical Manual of Mental Disorders (DSM-IV) criteria for dementia [30] and the National Institute of Neurological and Communication Disorders and Stroke-Alzheimer's Disease and Related Disorders Association criteria for probable AD [31]. Exclusion criteria in GARD were applied as follows: (1) illiteracy; (2) severe vision or hearing loss; (3) evidence of focal brain lesions on MRI other than suspected incipient AD; (4) any significant neurologic, medical, or neuropsychiatric disorders (e.g., depression or anxiety disorders) that could affect mental function; and (5) current use of psychoactive medications. The institutional review board of Chosun University Hospital and Chonnam National University approved the study. Written informed consent was obtained from each participant or his or her legal guardian. This study was conducted in accordance with the Declaration of Helsinki.

\section{CSF collection and analysis}

CSF was obtained by lumbar puncture with aseptic technique at the L3-L4 or L4-L5 intervertebral spinous process space, using a 22- or 21-gauge needle, and collected in Falcon polypropylene tubes (BD Biosciences, Franklin Lakes, NJ, USA). All CSF samples were analyzed using the Luminex 200 MAP system (INNO-BIA AlzBio3 for research-only reagents, Fujirebio, Ghent, Belgium). Samples were taken from the deep freezer 4 $\mathrm{h}$ before use and continuously kept on ice. After prewashing the 96 -well filter plate for vacuum with a $1 / 25$ diluted wash buffer, $25 \mu \mathrm{L}$ of each microsphere binding the corresponding ATN biomarker-specific capture antibodies (4D7A3, AT270, and AT120 for A $\beta_{1-42}$, $\mathrm{p}-\mathrm{Tau}_{181}$, and total tau protein [t-Tau], respectively) were bound with biotinylated monoclonal antibodies and capture antibodies (3D6 for $A \beta_{1-42}$, and HT7 for $\mathrm{p}-\mathrm{Tau}_{181}$ and t-Tau). Standard curves were constructed for ATN biomarkers using a sigmoidal curve-fitting, and the fluorescence intensity of the mean values for the duplicate samples were obtained with the concentration of ATN biomarkers. The cut-off values for the ATN biomarkers were determined using the Youden index method. Positive values were defined as follows (pg/mL): an "A" biomarker of $\mathrm{A} \beta_{1-42}<385.822$, "T" biomarker of p-Tau ${ }_{181}>41.881$, and "N" biomarker of $\mathrm{t}-\mathrm{Tau}>78.996$. Abnormal tau accumulation was designated based on " $\mathrm{T}$ " biomarker of $\mathrm{p}-\mathrm{Tau}_{181}>41.881$.

\section{Classification of $A$ and $T$ biomarker profiles}

There were 359 individuals who completed CSF analysis in the GARD database. Of these, we excluded candidates with evidence of focal brain lesions on MRI other than suspected incipient $\mathrm{AD}(n=8)$; any type of dementia other than $\mathrm{AD}(n=8)$; any significant neurologic, medical, or psychiatric disorders that could affect mental function $(n=19)$; no amyloid PET $(n=17)$; or inconsistent $\mathrm{A} \beta$ positivity between PET and CSF analysis ( $n$ $=48$ ); or no neuropsychological test scores available ( $n$ $=4$ ). In addition, individuals were excluded if the interval between lumbar puncture and neuropsychological assessment was $>6$ months $(n=38)$. Among the remaining 217 individuals 57 were $\mathrm{CN}$ older adults, 111 had $\mathrm{MCI}$, and 49 had AD.

Based on the National Institute on Aging and Alzheimer's Association research framework [1], we further excluded individuals with non- $\mathrm{AD}$ pathologic changes, that is, A-T $+(\mathrm{N})-(n=3,1 \mathrm{CN}, 1 \mathrm{MCI}$, and $1 \mathrm{AD}), \mathrm{A}-\mathrm{T}-$ $(\mathrm{N})+(n=10,2 \mathrm{CN}$ and $8 \mathrm{MCI})$, and $\mathrm{A}-\mathrm{T}+(\mathrm{N})+(n=6$, $3 \mathrm{CN}$ and $3 \mathrm{MCI}$ ). Individuals with Alzheimer's disease and concomitant suspected non-Alzheimer's pathologic changes, that is, $\mathrm{A}+\mathrm{T}-(\mathrm{N})+(n=6,2 \mathrm{CN}, 3 \mathrm{MCI}$, and 1 $\mathrm{AD})$, and individuals with normal AD biomarkers in the AD group, that is, A-T-(N)- $(n=7)$ were also excluded. Therefore, $49 \mathrm{CN}$ individuals, 96 with $\mathrm{MCI}$, and $40 \mathrm{AD}$ dementia were included in the final analysis (Fig. 1) (Supplementary Table S1).

The neurodegeneration biomarker $\mathrm{N}$ is not specific for $\mathrm{AD}$ and cannot define the AD continuum [1, 32]. Many other conditions, such as aging and cerebrovascular damage, can contribute to neurodegeneration [32-34]. Furthermore, $\mathrm{t}$-Tau shows high correlation with $\mathrm{p}$-tau [35] indicating little additive value of $\mathrm{t}$-Tau when making a diagnosis and prognosis of $\mathrm{AD}[36,37]$. Therefore, we categorized groups based only on A (based on the cutoff of $\mathrm{A} \beta_{1-42}$ ) and $\mathrm{T}$ (based on the cutoff of $\mathrm{p}-\mathrm{Tau}_{181}$ ) positivity. The participants were divided into three groups according to their $\mathrm{A}$ and $\mathrm{T}$ biomarker profiles: A-T-, $\mathrm{A}+\mathrm{T}-$, and $\mathrm{A}+\mathrm{T}+$.

\section{Clinical and neuropsychological assessments}

Information on participants' demographic characteristics and medical history were collected. The Geriatric Depression Scale [38] and Korean version of the Mini-Mental State Examination (K-MMSE) [39] were administered. In addition, a comprehensive neuropsychological assessment was performed. The psychomotor speed was assessed using trail making test part A (TMT A). The attention domain was assessed using the digit span forward and digit span backward tasks. The language domain was assessed using a shortened version of 


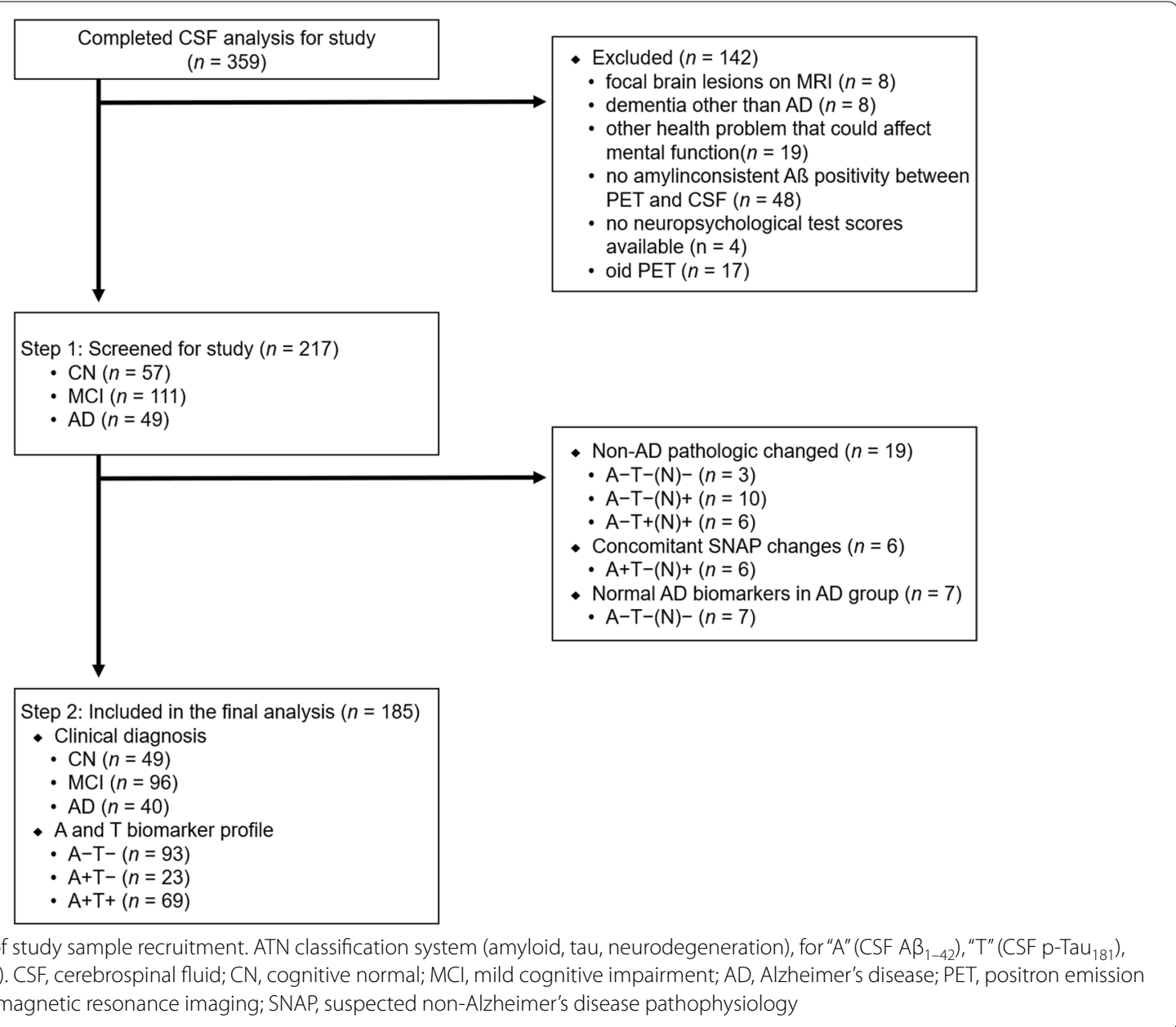

the Boston Naming Test (15-item version, form A). The visuospatial domain was assessed with the copying test from the Rey complex figure test (RCFT copy). The memory domain was assessed with six measures: the Seoul Verbal Learning Test (SVLT) immediate recall (SVLT imm), 20-min delayed recall (SVLT delayed), and yes-no recognition (SVLT rec), and the RCFT immediate recall (RCFT imm), 20-min delayed recall (RCFT delayed), and yes-no recognition (RCFT rec). For $61 \%(n=113)$ of participants, the Logical Memory (LM) subtest of the 4th edition of the Wechsler Memory Scale [40] was additionally administered. It consists of three parts: immediate recall (LMI), delayed recall (LMII), and delayed recognition (LM rec). Executive function was assessed using the animal fluency test, a phonemic fluency test, the Stroop test (color naming in color-word incongruent conditions), and TMT B.

\section{Determination of apolipoprotein $\varepsilon 4$ genotype}

The procedure for determining the apolipoprotein (APOE) genotype has been previously described [41].
Briefly, genomic DNA was extracted from buffy coats isolated from whole blood, and the APOE genotype was determined by single-nucleotide polymorphisms of rs429358 and rs7412. The APOE $\varepsilon 4$ positive genotype was assigned if at least one $\varepsilon 4$ allele was present.

\section{Imaging acquisition and processing}

MRI was performed using a 3.0 T MR scanner (Skyra, Siemens; 20-channel head coil; MRAGE sagittal view; TR $=2300 \mathrm{~ms} ; \mathrm{TE}=2.143 \mathrm{~ms} ; \mathrm{TI}=900 \mathrm{~ms} ; \mathrm{FA}=9^{\circ} ; \mathrm{FoV}=$ $256 \mathrm{~mm} \times 256 \mathrm{~mm}$, matrix $=320 \times 320$, and slice thickness $=0.8 \mathrm{~mm}$ ). MRI data from 11 participants were excluded from the analysis because they were scanned using 1.5 T. The volumes of cortical and subcortical structures were measured from each brain image using the standard recon-all processing pipeline of FreeSurfer version 5.3.0 (http://surfer.nmr.mgh.harvard.edu/). The automated reconstruction protocol has been described previously [42, 43]. We selected two regions of interest (ROIs) from the MTL including the hippocampus (HC) volume and entorhinal cortex (EC) thickness, and 
intracranial volume (ICV). Additionally, MRI ROIs outside MTL region including lingual gyrus, thalamus, and orbitofrontal area also obtained as control ROIs to confirm discriminative validity. ROIs were obtained using Aseg Atlas (https://surfer.nmr.mgh.harvard.edu/ftp/articles/fischl02-labeling.pdf) [44], and the Desikan-KillianyTourville atlas [45].

\section{Validation of the prediction model}

We tested the validity of the prediction model for abnormal tau accumulation, using an independent dataset. The validation dataset was obtained from the validation cohort of the Korean Brain Aging Study for Early Diagnosis and Prediction of AD (KBASE-V) [46]. We included CSF data and compatible clinical information, such as neuropsychological test scores and MRI, where available. The validation dataset consisted of $49 \mathrm{~A}-\mathrm{T}-, 5 \mathrm{~A}+\mathrm{T}-$, and $17 \mathrm{~A}+\mathrm{T}+$ (Supplementary Table S2).

\section{Statistical analysis}

Demographic characteristics and clinical information were compared among groups using separate one-way analyses of variance (ANOVA) and $\chi^{2}$ tests for continuous and categorical variables, respectively. MTL ROIs were compared among groups, controlling for ICV using analyses of covariance. Analyses of covariance were also performed to compare neuropsychological scores, controlling for age, education, and gender. We controlled demographic variables because neuropsychological test performance in Korean older adults strongly influenced by them [47]. To control for type I errors, a Bonferroni correction was carried out. When the results of the analyses of covariance were significant, a pairwise Bonferroni post hoc test was applied. Partial correlations between the p-Tau level and neuropsychological scores controlling for age, gender, education, APOE $\varepsilon 4$ positivity, K-MMSE, and $A \beta_{1-42}$ levels were performed. For neuropsychological scores that showed significant group difference, we performed hierarchical multivariable linear regression analysis. In the first step, age, gender, education, APOE $\varepsilon 4$ positivity, K-MMSE, and $A \beta_{1-42}$ levels were entered. These variables were considered as control variables; therefore, they were entered with "enter" method. Then, neuropsychological scores were entered with "stepwise selection" method in order to identify specific neuropsychological measures that associated with $\mathrm{p}$-Tau level. To test whether MTL atrophy (HC, EC) and other control ROIs mediated the association between these two, multiple mediation analysis was performed using 10,000 bootstrapping samples and $95 \%$ confidence intervals (CIs) [48]. Age, gender, education, and ICV were entered as covariates in the mediation analysis. Finally, a series of logistic regression analyses were conducted to develop a prediction model for abnormal tau accumulation. The predictors were entered into the models with adjustments for the demographic variables, and ICV was adjusted for MTL ROIs. To compare the predictability among the various models, we used the differences of $\log$ likelihood (-2LL). The -2LL is directly proportional to the contribution of variables to the separation of groups, and a smaller -2LL indicates better predictability of the model [49]. After selecting the optimal models, receiver operating characteristic (ROC) curve analysis was performed to examine the discrimination ability of the model. In addition, the prediction model was applied to an independent sample (validation dataset) to validate the model. Additional ROC curve analysis was performed if suggested models also sufficiently discriminate clinical status $(\mathrm{CN}$, $\mathrm{MCI}$, and $\mathrm{AD}$ dementia). These analyses were performed using SPSS version 25.0, and the PROCESS macro for SPSS (IBM Corporation, Armonk, NY, USA). A $p$-value less than 0.05 was considered statistically significant.

\section{Results}

\section{Participant characteristics}

Independent of the clinical diagnosis, 185 participants were divided into three groups based on the biomarker combination of $A$ and $T$ positivity. Ninety-three participants were classified as having normal AD biomarkers (A-T-), 23 were found to have Alzheimer's pathologic changes $(\mathrm{A}+\mathrm{T}-)$, and 69 had $\mathrm{AD}(\mathrm{A}+\mathrm{T}+)$. There were no group differences in age, education, gender, or subjective depression levels. APOE $\varepsilon 4$ carriers were more frequent in the $\mathrm{A}+\mathrm{T}$ - and $\mathrm{A}+\mathrm{T}+$ groups than in the $\mathrm{A}-\mathrm{T}$ - group $(p<0.001) . \mathrm{A} \beta_{1-42}$ levels were significantly lower in the $\mathrm{A}+\mathrm{T}$ - and $\mathrm{A}+\mathrm{T}+$ groups than in the $\mathrm{A}-\mathrm{T}$ - group $(p<$ $0.001)$. p-Tau and t-Tau were significantly higher in the $\mathrm{A}+\mathrm{T}+$ group than in the other two groups $(p<0.001)$. K-MMSE score and MTL ROIs values in the $\mathrm{A}+\mathrm{T}+$ group were significantly lower in $\mathrm{A}+\mathrm{T}+$ group than in $\mathrm{A}-\mathrm{T}$ - and $\mathrm{A}+\mathrm{T}-$, while there was no significant difference between A-T- and A+T- (Table 1). The mean time between CSF sampling and neuropsychological assessment was $2.75 \pm 1.58$ months and between CSF sampling and MRI scan was $2.32 \pm 2.37$ months.

\section{Neuropsychological characteristics}

Neuropsychological characteristics are shown in Table 2. RCFT imm, RCFT delayed, RCFT rec, and SVLT rec scores were significantly lower in the $\mathrm{A}+\mathrm{T}+$ group than in the A-T- and A+T- groups. SVLT imm and SVLT delayed scores were lower in the $\mathrm{A}+\mathrm{T}+$ group than in the A-T-group. RCFT copy score was lower in the $\mathrm{A}+\mathrm{T}+$ group than in the $\mathrm{A}+\mathrm{T}$ - group (Table 2). After Bonferroni correction, three memory scores from the RCFT and two scores from the SVLT remained 
Table 1 Demographic and clinical characteristics of study participants

\begin{tabular}{|c|c|c|c|c|}
\hline & A-/T- $(n=93)$ & $\mathrm{A}+/ \mathrm{T}-(n=23)$ & $\mathrm{A}+/ \mathrm{T}+(n=69)$ & $p$ value \\
\hline Age & $70.3 \pm 6.3$ & $71.3 \pm 6.5$ & $69.5 \pm 9.0$ & 0.606 \\
\hline Education & $10.4 \pm 5.0$ & $9.8 \pm 5.8$ & $9.1 \pm 4.6$ & 0.249 \\
\hline Female, $n(\%)$ & $46(49.5)$ & $13(56.5)$ & $36(52.2)$ & 0.820 \\
\hline APOE $\varepsilon 4$ carrier, ${ }^{a} n$ (\%) & $16(17.2)$ & $17(77.3)$ & $42(60.9)$ & $<0.001$ \\
\hline MMSE & $26.6 \pm 2.6$ & $25.0 \pm 4.5$ & $22.4 \pm 5.4^{*} t$ & $<0.001$ \\
\hline GDS & $10.6 \pm 6.9$ & $9.6 \pm 6.6$ & $10.6 \pm 6.5$ & 0.822 \\
\hline CN/MCl/AD, $n$ & $41 / 52 / 0$ & $6 / 12 / 5$ & $3 / 31 / 35$ & $<0.001$ \\
\hline $\mathrm{CDR}^{\mathrm{a}} 0, n(\%)$ & $41(44.1)$ & $7(30.4)$ & $3(4.3)$ & $<0.001$ \\
\hline $0.5, n(\%)$ & $52(55.9)$ & $15(65.2)$ & $43(62.3)$ & \\
\hline $1, n(\%)$ & $0(0.0)$ & $1(4.3)$ & $22(31.9)$ & \\
\hline \multicolumn{5}{|l|}{ CSF biomarkers (pg/ml) } \\
\hline$A \beta_{1-42}$ & $516.8 \pm 98.4$ & $224.5 \pm 104.4^{*}$ & $235.2 \pm 88.9^{*}$ & $<0.001$ \\
\hline p-Tau 181 & $29.3 \pm 5.5$ & $31.2 \pm 5.8$ & $60.2 \pm 13.2^{* t}$ & $<0.001$ \\
\hline t-Tau & $49.6 \pm 13.1$ & $48.6 \pm 16.0$ & $108.7 \pm 40.2^{* t}$ & $<0.001$ \\
\hline \multicolumn{5}{|l|}{ MTL ROIs ${ }^{b}$} \\
\hline L. HC & $3951 \pm 50$ & $3675 \pm 105$ & $3215 \pm 61^{*+}$ & $<0.001$ \\
\hline R. HC & $4202 \pm 57$ & $4002 \pm 118$ & $3498 \pm 69^{*} t$ & $<0.001$ \\
\hline L.EC & $3.28 \pm 0.04$ & $3.28 \pm 0.08$ & $2.79 \pm 0.05^{* \dagger}$ & $<0.001$ \\
\hline R. EC & $3.52 \pm 0.04$ & $3.52 \pm 0.09$ & $3.11 \pm 0.05^{*}+$ & $<0.001$ \\
\hline
\end{tabular}

Data are presented as means \pm standard deviations, unless specified otherwise. A and T classification system, for " $A$ " (based on the value of CSF A $\beta_{1-42}$ ), and " $T$ " (based on the value of CSF $\mathrm{p}-\mathrm{Tau}_{181}$ ). The following CSF thresholds were used: $385.822 \mathrm{pg} / \mathrm{mL}$ for $\mathrm{A} \beta_{1-42}$ and $41.881 \mathrm{pg} / \mathrm{mL}$ for $\mathrm{p}-\mathrm{Tau}{ }_{181}$

$A P O E$ apolipoprotein, K-MMSE Korean version of the Mini-Mental State Examination, GDS Geriatric Depression Scale, $C D R$ Clinical dementia rating, $A \beta_{1-42}$ amyloid $\beta_{(1-42)}, p-T_{a u}$, phosphorylated tau, $t$-Tau total tau protein, MTL medial temporal lobe, $R O I$ region of interest, $L$ left, $R$ right, $H C$ hippocampus volume, $E C$ entorhinal cortex thickness

${ }^{\text {a }}$ Missing data for one subject

b Missing data for 11 subjects

* Significantly different between the indicated group and the A-/T- group

${ }^{\dagger}$ Significantly different between the $A+/ T$ - and $A+/ T+$ groups

significant. However, no group differences were found in the test scores for psychomotor speed, attention, language, and executive function. A subset of participants who completed whole memory tests including LM (A-T$=80, \mathrm{~A}+\mathrm{T}-=14, \mathrm{~A}+\mathrm{T}+=19)$ also showed similar results on the RCFT and SVLT. In contrast, there were no differences in LM I, LM II, and LM rec scores among the groups (Table 2).

\section{Relationship between $\mathrm{p}$-Tau level and cognitive function}

Partial correlation of p-Tau level with neuropsychological scores controlling for demographic, K-MMSE, APOE $\varepsilon 4$ positivity, and $A \beta_{1-42}$ levels showed a significant association between visuospatial memory scores and p-Tau level (e.g., RCFT imm, $-0.285, p=0.007$; RCFT delayed, $-0.254, p=0.017$; and RCFT rec, $0.209, p=0.051$ ). For verbal memory scores, however, weaker (SVLT rec) or no associations (SVLT imm, SVLT delayed) were observed (Table 3). None of the other neuropsychological test scores showed a significant correlation with $\mathrm{p}$-Tau level. Hierarchical multiple linear regression analysis revealed that the RCFT delayed score was the only significant predictor for p-Tau level after controlling for demographic, K-MMSE, APOE $\varepsilon 4$ positivity, and $A \beta_{1-42}$ levels (Table 4 ). In addition, all three biomarkers were included in a regression model to test whether $A \beta_{1-42}$ and $t$-Tau levels also contribute to such memory measures. Both $\mathrm{p}$-Tau $(\beta=-0.329, p<0.0001$ for RCFT delayed; $\beta=-0.191, p=0.004$ for SVLT delayed) and $A \beta_{1-42}(\beta=0.163, p=0.027$ for RCFT delayed; $\beta=0.190, p=0.008$ for RCFT delayed) were significant, whereas t-Tau was insignificant (Supplementary table S3 and S4).

\section{Mediating role of MTL atrophy}

To test whether MTL atrophy plays a mediating role in the association between p-Tau and memory function, multiple mediation analyses were performed on delayed recall scores. The direct effect of p-Tau level on the RCFT delayed score was significant (effect $=-$ $0.1371, p<0.00001$ ), and the right HC-mediated (indirect) effect of $\mathrm{p}$-Tau level was significant for the RCFT 
Table 2 Group difference in neuropsychological test scores

\begin{tabular}{|c|c|c|c|c|c|}
\hline & $\mathrm{A}-\mathrm{T}^{-\mathrm{a}}$ & $A+/ T^{-b}$ & $\mathrm{~A}+/ \mathrm{T}+^{\mathrm{c}}$ & $p$ value & Post hoc \\
\hline \multicolumn{6}{|c|}{ Psychomotor speed } \\
\hline TMT A & $37.51 \pm 2.60$ & $31.40 \pm 5.00$ & $39.50 \pm 3.24$ & 0.395 & n.s \\
\hline \multicolumn{6}{|l|}{ Attention } \\
\hline DSF & $5.41 \pm 0.14$ & $5.67 \pm 0.27$ & $5.76 \pm 0.17$ & 0.278 & n.s \\
\hline DSB & $3.22 \pm 0.10$ & $3.67 \pm 0.19$ & $3.45 \pm 0.12$ & 0.083 & n.s \\
\hline \multicolumn{6}{|l|}{ Language } \\
\hline BNT & $43.63 \pm 0.98$ & $45.29 \pm 1.91$ & $42.62 \pm 1.22$ & 0.497 & n.s \\
\hline \multicolumn{6}{|c|}{ Visuospatial function } \\
\hline RCFT copy & $31.02 \pm 0.62$ & $33.14 \pm 1.20$ & $28.75 \pm 0.75$ & 0.006 & $b>c$ \\
\hline \multicolumn{6}{|l|}{ Memory } \\
\hline SVLT imm & $17.20 \pm 0.46$ & $15.71 \pm 0.89$ & $15.12 \pm 0.55$ & 0.020 & $a>c$ \\
\hline SVLT delayed & $4.81 \pm 0.22$ & $3.29 \pm 0.43$ & $2.71 \pm 0.27$ & $1.5 \mathrm{E}-7^{\dagger}$ & $a>b, c$ \\
\hline SVLT rec & $19.90 \pm 0.25$ & $19.26 \pm 0.48$ & $17.87 \pm 0.30$ & $1.1 \mathrm{E}-5^{\dagger}$ & $a, b>c$ \\
\hline LM I & $15.04 \pm 0.65$ & $13.50 \pm 1.54$ & $12.25 \pm 1.41$ & 0.200 & n.s \\
\hline LM II & $10.43 \pm 0.68$ & $9.38 \pm 1.60$ & $8.02 \pm 1.46$ & 0.343 & n.s \\
\hline LM rec & $19.25 \pm 0.51$ & $17.54 \pm 1.20$ & $16.51 \pm 1.09$ & 0.070 & n.s \\
\hline RCFT imm & $13.71 \pm 0.66$ & $12.56 \pm 1.27$ & $6.22 \pm 0.79$ & $2.8 \mathrm{E}-10^{+}$ & $a, b>c$ \\
\hline RCFT delayed & $13.49 \pm 0.65$ & $12.69 \pm 1.25$ & $5.66 \pm 0.79$ & $2.3 \mathrm{E}-11^{\dagger}$ & $a, b>c$ \\
\hline RCFT rec & $18.92 \pm 0.21$ & $18.48 \pm 0.40$ & $17.19 \pm 0.25$ & $6.0 E-6^{+}$ & $a, b>c$ \\
\hline \multicolumn{6}{|c|}{ Executive function } \\
\hline Fluency A & $13.35 \pm 0.43$ & $13.06 \pm 0.82$ & $11.70 \pm 0.51$ & 0.062 & n.s \\
\hline Fluency P & $21.44 \pm 0.85$ & $22.68 \pm 1.65$ & $20.15 \pm 1.06$ & 0.402 & n.s \\
\hline Stroop & $71.54 \pm 2.27$ & $63.13 \pm 4.37$ & $67.57 \pm 2.79$ & 0.201 & n.s \\
\hline TMT B & $68.12 \pm 5.16$ & $89.58 \pm 11.03$ & $75.09 \pm 7.11$ & 0.196 & n.s \\
\hline
\end{tabular}

Data are presented as means \pm standard deviations. $A$ and T classification system, for " $A$ " (based on the value of CSF A $\beta_{1-42}$ ), and "T" (based on the value of CSF $\mathrm{p}$-Tau $\left.{ }_{181}\right)$. The following CSF thresholds were used: $385.822 \mathrm{pg} / \mathrm{mL}$ for A $\beta_{1-42}$, and $41.881 \mathrm{pg} / \mathrm{mL}$ for $\mathrm{p}-\mathrm{Tau}_{181}$

TMT trail making test, DSF digit span forward, DSB digit span backward, BNT Boston naming test (15 item), RCFT copy Rey complex figure test copy score, SVLT imm Seoul verbal learning test immediate recall score, SVLT delayed SVLT delayed recall score, SVLT rec SVLT recognition score, LM I Logical Memory immediate recall score, $L M$ II Logical Memory, delayed recall score, $L M$ rec Logical Memory recognition score, RCFT imm RCFT immediate recall score, RCFT delayed RCFT delayed recall score, RCFT rec RCFT, recognition score, Fluency A fluency score for animal, Fluency P fluency score for 3 Korean letters, Stroop Stroop score for color naming in color-word in incongruent condition

${ }^{\mathrm{a}} \mathrm{A}-/ \mathrm{T}-$

${ }^{\mathrm{b}} \mathrm{A}+/ \mathrm{T}-$

${ }^{\mathrm{C}} \mathrm{A}+/ \mathrm{T}+$

${ }^{\dagger}$ Significant group difference at the Bonferroni corrected level, $p<0.0028$

delayed score (Fig. 2A). However, left HC did not mediate the association between p-Tau level and the RCFT delayed score. The direct effect of p-Tau level on the SVLT delayed score was also significant (effect $=-$ 0.0293, $p=0.0112$ ) and the left HC-mediated (indirect) effect of $\mathrm{p}$-Tau level was significant for the SVLT delayed score (Fig. 2B). Right HC did not mediate the association between the two. Bilateral EC showed no mediating effect on the association between p-Tau level and either of the two memory scores. Additionally, MRI ROIs outside MTL (lingual gyrus, thalamus, and orbitofrontal areas) showed no significant mediating role between p-Tau level and memory scores (Supplementary Table S5).

\section{Development of prediction models for abnormal tau accumulation}

For simplicity of the model, predictors were entered after demographic and ICV adjustments. In the first step, we tested one candidate model with RCFT delayed, which was based on the results of the hierarchical multiple linear regression analysis. RCFT delayed alone significantly predicted abnormal tau accumulation with $78.2 \%$ classification accuracy (Table 5, model A). In the second step, we tested several candidate models. Bilateral $\mathrm{HC}$ and bilateral EC were entered into each model (models B and C, respectively). Model A showed significantly smaller -2LL than the models with $\mathrm{HC}$ and $\mathrm{EC}$, demonstrating better predictability (Table 5 , models $\mathrm{B}$ and $\mathrm{C}$ ). In addition, 
Table 3 Partial correlations of p-Tau with neuropsychological scores

\begin{tabular}{|c|c|c|}
\hline \multirow[t]{2}{*}{ Neuropsychological tests } & \multicolumn{2}{|l|}{ p-Tau } \\
\hline & $r$ & $p$ value \\
\hline \multicolumn{3}{|l|}{ Psychomotor speed } \\
\hline TMT A & -0.019 & 0.862 \\
\hline \multicolumn{3}{|l|}{ Attention } \\
\hline DSF & 0.052 & 0.630 \\
\hline DSB & -0.205 & 0.056 \\
\hline \multicolumn{3}{|l|}{ Language } \\
\hline BNT & -0.001 & 0.991 \\
\hline \multicolumn{3}{|l|}{ Visuospatial function } \\
\hline RCFT copy & -0.185 & 0.084 \\
\hline \multicolumn{3}{|l|}{ Memory } \\
\hline SVLT imm & 0.014 & 0.898 \\
\hline SVLT delayed & -0.089 & 0.407 \\
\hline SVLT rec & -0.226 & 0.034 \\
\hline LM I & -0.134 & 0.213 \\
\hline LM II & -0.063 & 0.562 \\
\hline LM rec & -0.013 & 0.906 \\
\hline RCFT imm & -0.285 & 0.007 \\
\hline RCFT delayed & -0.254 & 0.017 \\
\hline RCFT rec & -0.209 & 0.051 \\
\hline \multicolumn{3}{|l|}{ Executive function } \\
\hline Fluency A & -0.070 & 0.519 \\
\hline Fluency $\mathrm{P}$ & 0.067 & 0.537 \\
\hline Stroop & 0.115 & 0.286 \\
\hline TMT B & -0.057 & 0.601 \\
\hline
\end{tabular}

Partial correlation was performed controlling for age, sex, education, Korean version of the Mini-Mental State Examination, apolipoprotein genotype, and amyloid- $\beta_{(1-42)}$

p-Tau phosphorylated tau, TMT trail making test, DSF digit span forward, DSB digit span backward, BNT Boston naming test (15 item), RCFT copy Rey complex figure test copy score, SVLT imm Seoul verbal learning test immediate recall score, SVLT delayed SVLT delayed recall score, SVLT rec SVLT recognition score, LM I Logical Memory immediate recall score, LM II Logical Memory, delayed recall score, $L M$ rec Logical Memory recognition score, RCFT imm RCFT immediate recall score, $R C F T$ delayed $\mathrm{RCFT}$ delayed recall score, $R C F T$ rec $\mathrm{RCFT}$, recognition score, Fluency $A$ fluency score for animal, Fluency $P$ fluency score for 3 Korean letters, Stroop Stroop score for color naming in color-word in incongruent condition

logistic regression with the forward conditional method was performed in order to develop optimal two candidate models among RCFT delayed, bilateral $\mathrm{HC}$, and bilateral EC. Consequently, RCFT delayed $(B=-1.314, p<$ 0.0001 , odds ratio $=0.269,95 \% \mathrm{CI}=0.161-0.448)$ and left EC $(B=-3.190, p<0.0001$, odds ratio $=0.041,95 \%$ $\mathrm{CI}=0.010-0.174)$ were selected, and they significantly predicted abnormal tau accumulation with $85.6 \%$ classification accuracy (Table 5, model D). The combination of RCFT delayed and left EC showed higher classification accuracy and significantly lower -2LL than model A. In the third step, left $\mathrm{HC}$ was added to model $\mathrm{D}$, but there was no significant -2LL difference between models $\mathrm{D}$ and E. Therefore, models A and D were finally selected. The prediction equation is as follows:

$$
\begin{aligned}
\text { Model A : } Y=-1.952 & +(-1.536 \times \text { RCFT delayed }) \\
\text { Model D : } Y=12.406 & +(-1.314 \times \text { RCFT delayed }) \\
& +(-3.190 \times \text { left EC })
\end{aligned}
$$

As shown in Table 6, the area under the curve (AUC) of the ROC curve using prediction models $A$ and $D$ were 0.872 and 0.921 , respectively, indicating good discrimination between $\mathrm{T}$ - and $\mathrm{T}+$. These equations were applied to the validation dataset. The AUC of the ROC curve in the validation dataset for models $\mathrm{A}$ and $\mathrm{D}$ were 0.879 and 0.891 , respectively (Table 6). Additional ROC curve analysis on clinical status revealed that our models also discriminate between $\mathrm{CN}$ and $\mathrm{AD}$ (AUC $=0.956$ for model $\mathrm{D}$ ) and between $\mathrm{MCI}$ and $\mathrm{AD}$ (AUC $=0.825$ for model D) (Supplementary Table S6).

\section{Discussion}

This study investigated whether tau accumulation is associated with particular cognitive impairment across the AD continuum and whether MTL atrophy mediates this association. We also developed and validated optimal prediction models for abnormal tau accumulation. We found an association between decreased visuospatial delayed memory performance and increased p-Tau levels. Temporal lobe neurodegeneration mediates the association between the two. Prediction models with visuospatial delayed memory and EC thickness for abnormal tau accumulation were validated. Preliminary findings from the current study might provide important insights into the association between tau pathology and cognitive symptoms in the AD continuum.

Cognitive impairment is a core clinical feature of the AD continuum. Given that tau protein accumulation, not $A \beta$ burden, is highly associated with $A D$ related clinical symptoms [13-15], understanding the independent influence of tau accumulation on cognitive function is important for early detection and developing interventions in AD. This study found tauassociated decreased episodic memory performance that was independent of other relevant variables such as $A \beta$ pathology level, APOE $\varepsilon 4$ positivity, and global cognitive function. Additional analyses to test relative contributions of $A \beta_{1-42}, p$-Tau and t-Tau on the memory scores revealed that $\mathrm{p}$-Tau was the most significantly associated with memory measures (Supplementary table S3 and S4). These findings are in line with the notion that pathologic tau is a primary factor in AD-related memory change [13]. Most notably, such associations were observed mainly in the visuospatial 
Table 4 Hierarchical multiple linear regression analysis for neuropsychological tests associated with p-Tau

\begin{tabular}{|c|c|c|c|c|c|c|}
\hline \multirow[t]{2}{*}{ Independent variables } & \multicolumn{3}{|l|}{ Step $1^{a}$} & \multicolumn{3}{|l|}{ Step $2^{b}$} \\
\hline & $B$ & $S E B$ & $\beta$ & $B$ & $S E B$ & $\beta$ \\
\hline Constant & $90.685^{* *}$ & 12.332 & & $82.134^{* *}$ & 11.764 & \\
\hline Sex & 2.953 & 2.435 & 0.087 & 4.139 & 2.309 & 0.122 \\
\hline Age & -0.244 & 0.149 & -0.106 & -0.274 & 0.140 & -0.119 \\
\hline Education & -0.220 & 0.252 & -0.065 & -0.146 & 0.238 & -0.043 \\
\hline APOE $\varepsilon 4$ & 1.119 & 2.456 & 0.033 & 0.949 & 2.316 & 0.028 \\
\hline K-MMSE & $-0.680^{*}$ & 0.290 & -0.180 & -0.146 & 0.295 & -0.039 \\
\hline$A \beta_{1-42}$ & $-0.042^{* *}$ & 0.007 & -0.423 & $-0.031^{* *}$ & 0.007 & -0.314 \\
\hline RCFT delayed & & & & $-0.754^{* *}$ & 0.158 & -0.363 \\
\hline
\end{tabular}

K-MMSE Korean version of the Mini-Mental State Examination, $A \beta_{1-42}$ amyloid $\beta_{(1-42),} p$-Tau phosphorylated tau, RCFT delayed Rey complex figure test delayed recall score

${ }^{a} R^{2}=0.305, F=12.664^{* *}$

${ }^{\mathrm{b}} R^{2}=0.386, \Delta R^{2}=0.081, F=15.441, \Delta F=22.642^{* *}$

${ }^{*} p<0.05$

${ }^{* *} p<0.001$

\section{(A)}

$-0.1416(0.0532),[-0.2473,-0.0359]$

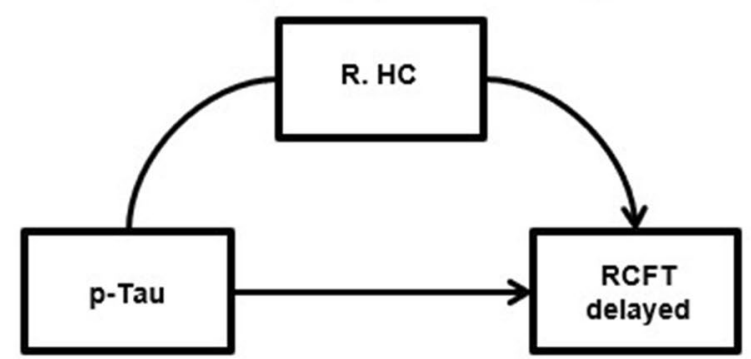

(B)

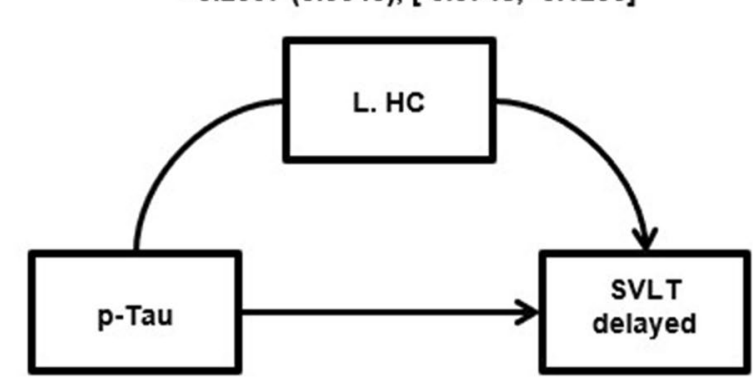

Fig. 2 Hippocampus atrophy mediates the relationship between $p$-Tau level and memory scores. A The mediation of the relationship between p-Tau level and RCFT delayed score by right hippocampus volume. B The mediation of the relationship between p-Tau level and SVLT delayed score by left hippocampus volume. Values: Effect (BootSE), [BootLLCI, BootULCI]. p-Tau, phosphorylated tau; RCFT, delayed Rey complex figure test delayed recall score; SVLT, delayed Seoul verbal learning test delayed recall score; R, HC right hippocampus volume; L, HC left hippocampus volume

Table 5 Logistic regression analysis to select appropriate models for abnormal tau accumulation prediction

\begin{tabular}{lllllll}
\hline Models $^{\mathbf{a}}$ & $\begin{array}{l}\text { Classification } \\
\text { accuracy (\%) }\end{array}$ & $\mathbf{- 2 ~ L L}$ & $\mathbf{X}^{\mathbf{2}}$ & $\mathbf{d f}$ & $\boldsymbol{p}$ value & Significant test for -2LL difference \\
\hline $\begin{array}{l}\text { One candidate model } \\
\quad \text { Model A: RCFT delayed }\end{array}$ & 78.2 & 150.171 & 76.472 & 1 & $<0.001$ & \\
$\begin{array}{l}\text { Two-candidate model } \\
\quad\end{array}$ & 79.3 & 161.510 & 65.133 & 2 & $<0.001$ & Model A vs. B: $p=0.0009$ \\
$\quad \begin{array}{l}\text { Model B: R.HC + L. HC } \\
\quad \text { Model C: R.EC + L. EC }\end{array}$ & 75.9 & 162.299 & 64.344 & 2 & $<0.001$ & Model A vs. C: $p=0.0005$ \\
$\quad \begin{array}{l}\text { Model D: RCFT delayed + L.EC } \\
\text { Three-candidate model }\end{array}$ & 85.6 & 123.479 & 103.163 & 2 & $<0.001$ & Model A vs. D: $p<0.001$ \\
$\quad$ Model E: RCFT delayed + L.HC + L. EC & 88.5 & 120.828 & 105.814 & 3 & $<0.001$ & Model D vs. E: $p=0.1069$ \\
\hline
\end{tabular}

RCFT delayed Rey complex figure test delayed recall score, $R$. HC right hippocampus volume, $L H C$ left hippocampus volume, L. EC left entorhinal cortex thickness a Variables were adjusted for age, sex, and education, and ICV adjustment was added for HC volume and EC thickness 
Table 6 AUCs of models A and D in study dataset and validation dataset

\begin{tabular}{|c|c|c|c|c|}
\hline \multirow[t]{2}{*}{ Predictor } & \multicolumn{2}{|l|}{ Study dataset } & \multicolumn{2}{|c|}{ Validation dataset } \\
\hline & AUC (SE) & $95 \% \mathrm{Cl}$ & AUC (SE) & $95 \% \mathrm{Cl}$ \\
\hline $\begin{array}{l}\text { Model A: RCFT delayed } \\
\qquad Y=-1.952+(-1.536 \times R C F\end{array}$ & $0.872(0.028)^{*}$ & $0.818-0.925$ & $0.879(0.046)^{*}$ & $0.788-0.969$ \\
\hline Model D: RCFT delayed +L. EC & $0.921(0.024)^{*}$ & $0.874-0.969$ & $0.891(0.052)^{*}$ & $0.789-0.993$ \\
\hline \multicolumn{5}{|c|}{$Y=12.406+(-1.314 \times$ RCFT delayed $)+(-3.190 \times \mathrm{L} . \mathrm{EC})$} \\
\hline
\end{tabular}

$R C F T$ delayed Rey complex figure test delayed recall score, $L$. EC left entorhinal cortex thickness

${ }^{*} p<0.001$

modality of episodic memory. Our result is largely consistent with those of previous studies showing associations between tau pathology and episodic memory $[8,11,16,17]$. However, few studies have included visuospatial episodic memory measures or controlled for other possible confounding variables, such as $A \beta$ pathology or APOE genotype. The current results extend those of previous studies in that we included comprehensive neuropsychological assessments covering major cognitive domains; therefore, we preliminarily found tau-associated visuospatial memory impairment. In comparison with the verbal memory test score (SVLT delayed score), the visuospatial memory test score (RCFT delayed score) has a wider range of scores (SVLT delayed 0-12 vs. RCFT delayed 0-36) and higher test difficulty (recall of a simple 12-word list vs. recall of both correct positions and visually complex figures of 18 items). Therefore, we performed additional analyses on the subgroup who completed more difficult verbal memory tests, including LM testing, to rule out the possibility of the contribution of features of the neuropsychological test tool itself to the current results. Because the LM test has much higher difficulty (two stories with 25 units each) and a wider score range (0-50) than the SVLT delayed test, we assumed that the LM II test (the delayed recall part) might comparable the RCFT delayed test. We found that the LM II score had no significant association with the p-Tau level, but the RCFT delayed score did from the subgroup analysis. However, the results should be interpreted with caution, because LM II test were only available for a subset of participants. Only two previous studies included visuospatial modality of memory test $[11,17]$, and their results were in line with ours, suggesting a stronger association with visuospatial episodic memory than with verbal modality. Animal studies have also found that tau hyperphosphorylated mice show impaired spatial learning ability [50]. Our preliminary findings suggest that tau accumulation underlies episodic memory impairments, especially visuospatial modality.
On the other hand, we did not find any link between p-Tau and other cognitive domains, such as executive function, attention, and language. This may be partly due to the considerably milder clinical severity in our study sample. Of all the participants in our study, $59.5 \%$ had a CDR of 0.5 , and only $12 \%$ had a CDR of 1 . Tau associations with the non-memory domain may appear at a later stage of $\mathrm{AD}$. Inclusion of more progressed cases of $\mathrm{AD}$ would provide a clearer picture of this issue.

Brain atrophy could be at least partially attributable to downstream of tau pathology [22, 25]. We focused on MTL areas because these regions are vulnerable to the initial tau pathology process [51]. It is highly unlikely that MTL atrophy in our dataset was caused by other etiology other than $\mathrm{AD}$, because we exclude individuals with potential non-AD pathologic changes. Mediation analysis revealed that $\mathrm{HC}$ volume, not EC thickness, mediated the association between $\mathrm{p}$-Tau levels and memory performance. Notably, the mediation effect of $\mathrm{HC}$ volume was observed in a modality-specific manner. Right HC-mediated indirect effect was found in visuospatial delayed memory performance, while left $\mathrm{HC}$ volume-mediated indirect effect was found in verbal delayed memory performance. Together, previous reports $[26,52,53]$ and our findings suggest that although various processes could influence brain atrophy, tau accumulation could partly lead to neuronal loss in $\mathrm{HC}$, and consequently cause episodic memory problems.

CSF tau accumulation precede cognitive decline $[2,26]$. Sometimes, cognitive profiles were not sensitive enough to define AD [1] at earlier stage of disease. Prediction of abnormal tau accumulation may provide prognosis even before cognitive deficits were clinically apparent or when cognitive changes were so subtle that it cannot captured by cognitive profile. Although tau accumulation is clinically valuable information, measuring it is not always feasible, especially in community-based settings. However, no studies have demonstrated a prediction model for abnormal tau accumulation. Therefore, we proposed prediction models to be used as a screening tool in a practical way, and validated them in an independent sample. 
Our results from a series of logistic regression analyses showed that the RCFT delayed score alone could predict abnormal tau accumulation efficiently. Moreover, the "RCFT delayed score only" model showed better predictability than models with MTL ROIs. This result provides preliminary evidence that abnormal tau accumulation might be more sensitively detected by the lower visuospatial memory performance than by MTL atrophy. This may be plausible, especially when people are in the early stages of clinical AD, as in our study sample. RCFT can be administered simply with pencil and paper and has reported to be clinically useful in geriatric population $[27,54,55]$. However, the test has a rather complicated scoring system and strongly influenced by age, gender, and intelligence or education $[54,56]$. Therefore, the test should be carefully scored and interpreted based on normative information.

Furthermore, the combination of RCFT delayed score and left EC thickness revealed an additional effect, resulting in higher classification accuracy than the RCFT delayed score alone. In line with our result, left side of EC was reported to be thinner in $\mathrm{MCI}$ group than $\mathrm{CN}$ group [57], or thinner in progressive (to $\mathrm{AD}$ ) $\mathrm{MCI}$ group than stable MCI group [58]. The ROC curve analysis using these two prediction equations demonstrated significant discrimination ability between $\mathrm{T}$ positive and $\mathrm{T}$ negative individuals in the independent validation sample as well as in the current study sample. Additionally, the suggested models also seem to be useful to discriminate $\mathrm{AD}$ from $\mathrm{CN}$ or $\mathrm{MCI}$, but not $\mathrm{MCI}$ from $\mathrm{CN}$.

Tau accumulation can be evaluated by either CSF or PET methods. In the current study we adopted CSF method, because CSF analysis can straightforwardly assess in vivo AD pathophysiology [59]. Specifically, elevated $\mathrm{p}$-Tau level is a direct biomarker of fibrillar tau. It best reflects the pathologic state at the time of the test that is associated with AD core pathology. CSF p-Tau denotes an ongoing active pathologic state and detects earlier pathological changes than PET $[2,60,61]$. However, it is worth mentioning that tau PET can also play a role as a state marker for cognitive decline in $\mathrm{AD}[8$, 62]. Tau PET measures the magnitude of pathological tau accumulation over time [1].

\section{Limitations}

Despite its significant implications, the current study has limitations and future directions to be discussed. First, this study used a cross-sectional design; therefore, the results should be interpreted with caution. Further studies with longitudinal follow-up could provide a better understanding of the actual cognitive consequences of abnormal tau accumulation. Second, sample size in the $\mathrm{A}+/ \mathrm{T}$ - subgroups (including validation sample) and in $\mathrm{CN}$ individuals with $\mathrm{AD}$ pathology-positive, i.e., preclinical stage, are very small. Larger sample size in such subgroups could shed more light on the association between abnormal tau accumulation and cognitive function in the initial process of the disease and provide more clinical implication. Lastly, our data provide preliminary evidence that visuospatial episodic memory tests could sensitively reflect abnormal tau accumulation. Given that verbal episodic memory tests are mainly used and the visuospatial tests are far less used in the field, it is certainly one area for additional research that could enhance the understanding of the nature of visuospatial episodic memory

\section{Conclusions}

In conclusion, it is crucial to identify sensitive cognitive measures that capture subtle cognitive impairment associated with underlying pathological changes. Preliminary findings from the current study might suggest that abnormal tau accumulation underlies episodic memory impairment, particularly visuospatial modality, in the AD continuum. Suggested models are potentially useful in predicting tau pathology and might be utilized practically in the field.

\section{Abbreviations}

AD: Alzheimer's disease; APOE: The apolipoprotein; AUC: The area under the curve; $A \beta$ : Amyloid-beta; $C D R$ : A clinical dementia rating; $C N$ : Cognitively normal; CSF: Cerebrospinal fluid; EC: Entorhinal cortex; GARD: The Gwanju Alzheimer's Disease and Related Dementia; HC: Hippocampus; ICV: Intracranial volume; KBASE-V: The Korean Brain Aging Study for Early Diagnosis and Prediction of AD; K-MMSE: Korean version of the Mini-Mental State Examination; LM: The Logical Memory; LM rec: Delayed recognition; LMI: Immediate recall; LMII: Delayed recall; MCI: Mild cognitive impairment; MRI: Magnetic resonance imaging; MTL: The medial temporal lobe; NFT: Neurofibrillary tangles; PET: Positron emission tomography; p-Tau: Phosphorylated tau; RCFT copy: The Rey complex figure test; RCFT delayed: The RCFT immediate recall 20-min delayed recall; RCFT imm: The RCFT immediate recall; RCFT rec: The RCFT immediate recall yes-no recognition; ROC: Receiver operating characteristic; SVLT: The Seoul Verbal Learning Test; SVLT delayed: The Seoul Verbal Learning Test 20-min delayed recall; SVLT imm: The Seoul Verbal Learning Test immediate recall; SVLT rec: The Seoul Verbal Learning Test yes-no recognition; TMT: Trail making tests; t-Tau: Total tau protein.

\section{Supplementary Information}

The online version contains supplementary material available at https://doi. org/10.1186/s13195-021-00909-1.

Additional file 1: Supplemental Table S1. Demographic and clinical characteristics of clinical diagnosis subgroups. Data are presented as means \pm standard deviations, unless specified otherwise. A and $T$ classification system, for " $A$ " (based on the value of CSF A $\beta_{1-42}$ ), and " $T$ " (based on the value of CSF $\left.p-T_{a u}{ }_{181}\right)$. The following CSF thresholds were used: $385.822 \mathrm{pg} / \mathrm{mL}$ for $A \beta_{1-42}$, and $41.881 \mathrm{pg} / \mathrm{mL}$ for $\mathrm{p}-T_{a u}{ }_{181}$. Supplemental Table S2. Clinical characteristics in validation data set. Data are presented as means \pm standard deviation, unless specified otherwise. The following CSF thresholds were used: $385.822 \mathrm{pg} / \mathrm{mL}$ for $A \beta_{1-42}$, and $41.881 \mathrm{pg} / \mathrm{mL}$ for $\mathrm{p}-\mathrm{Tau}_{181}$. Supplemental Table S3. Partial correlations of $A \beta_{1-42}$. and 
t-Tau with neuropsychological scores. Partial correlation was performed controlling for age, sex, education, Korean version of the Mini-Mental State Examination, apolipoprotein genotype, and CSF biomarkers. Supplemental Table S4. Multiple linear regression analysis with stepwise selection. Multiple linear regression was performed controlling for age, sex, education, Korean version of the Mini-Mental State Examination, and apolipoprotein genotype. Supplemental Table S5. Mediation analysis of MRI control regions of interests. Data are presented as effect (BootSE), [BootLLCI, BootULCI]. Supplemental Table S6. AUCs of models A and D in $\mathrm{CN}, \mathrm{MCl}$, and $\mathrm{AD}$ groups.

\section{Acknowledgements}

Not applicable.

\section{Authors' contributions}

KHL, EHS and HJL designed of the research. HJL, EHS, HJY, KYC, JJL, and JYP undertook the statistical analysis. $\mathrm{KHL}, \mathrm{HJL}, \mathrm{EHS}$, and $\mathrm{HJY}$ interpreted the data and wrote the draft of the manuscript. BCK, HWK, and SHC collected the data. KYC, JJL, JYP, BCK, HWK, and SHC critically revised the manuscript. The authors have read and approved the final manuscript.

\section{Funding}

This study was supported by KBRI basic research program through Korea Brain Research Institute funded by Ministry of Science and ICT (21-BR-03-05), the Original Technology Research Program for Brain Science of the National Research Foundation (NRF) funded by the Korean government, MSIT (NRF-2014M3C7A1046041 and NRF-2016M3C7A1905469), the Brain Convergence Research Program of the NRF funded by the Ministry of Science and ICT (NRF2020M3E5D2A01084721) and a Basic Science Research Program through the NRF of Korea (NRF-2020R1F1A1052932)

\section{Availability of data and materials}

The datasets analyzed for the current study are not publicly available but are available from the corresponding author on reasonable request.

\section{Declarations}

\section{Ethics approval and consent to participate}

The institutional review board of Chosun University Hospital (CHOSUN 201312-018-068) and Chonnam National University (CNUH-2019-279) approved this study. Written informed consent was obtained from each participant or his or her legal guardian.

\section{Consent for publication}

Not applicable.

\section{Competing interests}

The authors declare that they have no competing interests.

\section{Author details}

${ }^{1}$ Gwangju Alzheimer's Disease and Related Dementia Cohort Research Center, Chosun University, 61452 Gwangju, Republic of Korea. ${ }^{2}$ Premedical Science, College of Medicine, Chosun University, Gwangju 61452, Republic of Korea. ${ }^{3}$ Department of Integrative Biological Science, Chosun University, Gwangju 61452, Republic of Korea. ${ }^{4}$ Department of Neuropsychiatry, College of Medicine, Chosun University, Gwangju 61452, Republic of Korea. ${ }^{5}$ Department of Public Health Sciences, Graduate School of Public Health, Seoul National University, Seoul 08826, Republic of Korea. ${ }^{6}$ Department of Neurology, Inha University School of Medicine, Incheon 22212, Republic of Korea. ${ }^{7}$ Department of Neurology, Chosun University Hospital, Gwangju 61452, Republic of Korea. ${ }^{8}$ Department of Neurology, Chonnam National University Medical School, Gwangju 61469, Republic of Korea. ${ }^{9}$ Department of Biomedical Science, Chosun University, Gwangju 61452, Republic of Korea. ${ }^{10}$ Aging Neuroscience Research Group, Korea Brain Research Institute, Daegu 41062 , Republic of Korea. ${ }^{11}$ Neurozen Inc., Seoul 06236, Republic of Korea.
Received: 21 June 2021 Accepted: 28 September 2021

Published online: 09 October 2021

\section{References}

1. Jack CR Jr, Bennett DA, Blennow K, Carrillo MC, Dunn B, Haeberlein SB, et al. NIA-AA research framework: toward a biological definition of Alzheimer's disease. Alzheimers Dement. 2018;14(4):535-62. https://doi.org/10. 1016/j.jalz.2018.02.018.

2. Jack CR Jr, Knopman DS, Jagust WJ, Petersen RC, Weiner MW, Aisen PS, et al. Tracking pathophysiological processes in Alzheimer's disease: an updated hypothetical model of dynamic biomarkers. Lancet Neurol. 2013;12(2):207-16. https://doi.org/10.1016/S1474-4422(12)70291-0.

3. Fagan AM, Roe CM, Xiong C, Mintun MA, Morris JC. Holtzman DM Cerebrospinal fluid tau/beta-amyloid(42) ratio as a prediction of cognitive decline in nondemented older adults. Arch Neurol. 2007;64(3):343-9. https://doi.org/10.1001/archneur.64.3.noc60123.

4. Mattsson N, Zetterberg H, Hansson O, Andreasen N, Parnetti L, Jonsson $\mathrm{M}$, et al. CSF biomarkers and incipient Alzheimer disease in patients with mild cognitive impairment. JAMA. 2009;302(4):385-93. https://doi.org/10. 1001/jama.2009.1064

5. Delmotte K, Schaeverbeke J, Poesen K. Vandenberghe R Prognostic value of amyloid/tau/neurodegeneration (ATN) classification based on diagnostic cerebrospinal fluid samples for Alzheimer's disease. Alzheimers Res Ther. 2021;13(1):84. https://doi.org/10.1186/s13195-021-00817-4.

6. Huber CM, Yee C, May T, Dhanala A. Mitchell CS Cognitive decline in preclinical Alzheimer's disease: amyloid-beta versus tauopathy. J Alzheimers Dis. 2018;61 (1):265-81. https://doi.org/10.3233/JAD-170490.

7. Bierer LM, Hof PR, Purohit DP, Carlin L, Schmeidler J, Davis KL, et al. Neocortical neurofibrillary tangles correlate with dementia severity in Alzheimer's disease. Arch Neurol. 1995;52(1):81-8. https://doi.org/10. 1001/archneur.1995.00540250089017.

8. Lowe VJ, Bruinsma TJ, Wiste HJ, Min HK, Weigand SD, Fang P, et al. Crosssectional associations of tau-PET signal with cognition in cognitively unimpaired adults. Neurology. 2019;93(1):e29-39. https://doi.org/10. 1212/WNL.0000000000007728.

9. Ossenkoppele R, Smith R, Ohlsson T, Strandberg O, Mattsson N, Insel PS, et al. Associations between tau, Abeta, and cortical thickness with cognition in Alzheimer disease. Neurology. 2019;92(6):e601-12. https://doi.org/ 10.1212/WNL.0000000000006875.

10. Sperling RA, Mormino EC, Schultz AP, Betensky RA, Papp KV, Amariglio $\mathrm{RE}$, et al. The impact of amyloid-beta and tau on prospective cognitive decline in older individuals. Ann Neurol. 2019;85(2):181-93. https://doi. org/10.1002/ana.25395.

11. Maass A, Lockhart SN, Harrison TM, Bell RK, Mellinger T, Swinnerton $K$, et al. Entorhinal tau pathology, episodic memory decline, and neurodegeneration in aging. J Neurosci. 2018;38(3):530-43. https://doi.org/10. 1523/JNEUROSCI.2028-17.2017.

12. Kester MI, van der Vlies AE, Blankenstein MA, Pijnenburg YA, van Elk EJ, Scheltens $\mathrm{P}$, et al. CSF biomarkers predict rate of cognitive decline in Alzheimer disease. Neurology. 2009;73(17):1353-8. https://doi.org/10.1212/ WNL.0b013e3181bd8271.

13. Brier MR, Gordon B, Friedrichsen K, McCarthy J, Stern A, Christensen J, et al. Tau and Abeta imaging, CSF measures, and cognition in Alzheimer's disease. Sci TransI Med. 2016;8(338):338ra366. https://doi.org/10.1126/ scitranslmed.aaf2362.

14. Roberson ED, Scearce-Levie K, Palop JJ, Yan F, Cheng IH, Wu T, et al. Reducing endogenous tau ameliorates amyloid beta-induced deficits in an Alzheimer's disease mouse model. Science. 2007;316(5825):750-4. https://doi.org/10.1126/science.1141736.

15. Bloom GS. Amyloid-beta and tau: the trigger and bullet in Alzheimer disease pathogenesis. JAMA Neurol. 2014;71(4):505-8. https://doi.org/10. 1001/jamaneurol.2013.5847.

16. Okafor M, Nye JA, Shokouhi M, Shaw LM, Goldstein F. Hajjar I 18F-Flortaucipir PET associations with cerebrospinal fluid, cognition, and neuroimaging in mild cognitive impairment due to Alzheimer's disease. J Alzheimers Dis. 2020;74(2):589-601. https://doi.org/10.3233/ JAD-191330. 
17. Pettigrew C, Soldan A, Moghekar A, Wang MC, Gross AL, O'Brien R, et al. Relationship between cerebrospinal fluid biomarkers of Alzheimer's disease and cognition in cognitively normal older adults. Neuropsychologia. 2015:7863-72. https://doi.org/10.1016/j.neuropsychologia.2015.09.024.

18. Timmers $\mathrm{M}$, Tesseur I, Bogert J, Zetterberg H, Blennow K, BorjessonHanson A, et al. Relevance of the interplay between amyloid and tau for cognitive impairment in early Alzheimer's disease. Neurobiol Aging. 2019:79131-41. https://doi.org/10.1016/j.neurobiolaging.2019.03.016.

19. Snitz BE, Tudorascu DL, Yu Z, Campbell E, Lopresti BJ, Laymon CM, et al. Associations between NIH toolbox cognition battery and in vivo brain amyloid and tau pathology in non-demented older adults. Alzheimers Dement (Amst). 2020;12(1):e12018. https://doi.org/10.1002/dad2.12018.

20. Li G, Millard SP, Peskind ER, Zhang J, Yu CE, Leverenz JB, et al. Crosssectional and longitudinal relationships between cerebrospinal fluid biomarkers and cognitive function in people without cognitive impairment from across the adult life span. JAMA Neurol. 2014;71(6):742-51. https:// doi.org/10.1001/jamaneurol.2014.445.

21. Bos I, Vos SJB, Jansen WJ, Vandenberghe R, Gabel S, Estanga A, et al. Amyloid-beta, tau, and cognition in cognitively normal older individuals: examining the necessity to adjust for biomarker status in normative data. Front Aging Neurosci. 2018;10193. https://doi.org/10.3389/fnagi.2018 00193.

22. Knopman DS, Lundt ES, Therneau TM, Vemuri P, Lowe VJ, Kantarci K, et al. Entorhinal cortex tau, amyloid-beta, cortical thickness and memory performance in non-demented subjects. Brain. 2019;142(4):1148-60. https:// doi.org/10.1093/brain/awz025.

23. Braak H, Alafuzoff I, Arzberger T, Kretzschmar H. Del Tredici K Staging of Alzheimer disease-associated neurofibrillary pathology using paraffin sections and immunocytochemistry. Acta Neuropathol. 2006;112(4):389404. https://doi.org/10.1007/s00401-006-0127-z.

24. Jack CR Jr, Wiste HJ, Schwarz CG, Lowe VJ, Senjem ML, Vemuri P, et al. Longitudinal tau PET in ageing and Alzheimer's disease. Brain. 2018;141(5):1517-28. https://doi.org/10.1093/brain/awy059.

25. Das SR, Xie L, Wisse LEM, Ittyerah R, Tustison NJ, Dickerson BC, et al. Longitudinal and cross-sectional structural magnetic resonance imaging correlates of AV-1451 uptake. Neurobiol Aging. 2018:6649-58. https://doi. org/10.1016/j.neurobiolaging.2018.01.024.

26. Bejanin A, Schonhaut DR, La Joie R, Kramer JH, Baker SL, Sosa N, et al. Tau pathology and neurodegeneration contribute to cognitive impairment in Alzheimer's disease. Brain. 2017;140(12):3286-300. https://doi.org/10. 1093/brain/awx243.

27. Seo EH, Kim H, Choi KY, Lee KH. Choo IH Pre-mild cognitive impairment: can visual memory predict who rapidly convert to mild cognitive impairment? Psychiatry Investig. 2018;15(9):869-75. https://doi.org/10.30773/pi. 2018.07.29.1.

28. Chung JY, Yoon HJ, Kim H, Choi KY, Lee JJ, Lee KH, et al. Reversion from mild cognitive impairment to normal cognition: false-positive error or true restoration thanks to cognitive control ability? Neuropsychiatr Dis Treat. 2019:153021-32. https://doi.org/10.2147/NDT.S223958.

29. Winblad B, Palmer K, Kivipelto M, Jelic V, Fratiglioni L, Wahlund LO, et al. Mild cognitive impairment--beyond controversies, towards a consensus: report of the International Working Group on Mild Cognitive Impairment. J Intern Med. 2004;256(3):240-6. https://doi.org/10.1111/j.1365-2796. 2004.01380.x.

30. American Psychiatric Association. Diagnostic and statistical manual of mental disorders. Washington, DC: American Psychiatric Association Press: 1994

31. McKhann G, Drachman D, Folstein M, Katzman R, Price D. Stadlan EM Clinical diagnosis of Alzheimer's disease: report of the NINCDS-ADRDA Work Group under the auspices of Department of Health and Human Services Task Force on Alzheimer's Disease. Neurology. 1984;34(7):939-44. https://doi.org/10.1212/WNL.34.7.939.

32. Jack CR Jr, Bennett DA, Blennow K, Carrillo MC, Feldman HH, Frisoni GB, et al. A/T/N: an unbiased descriptive classification scheme for Alzheimer disease biomarkers. Neurology. 2016;87(5):539-47. https://doi.org/10. 1212/WNL.0000000000002923.

33. Kovacs GG, Milenkovic I, Wohrer A, Hoftberger R, Gelpi E, Haberler C, et al. Non-Alzheimer neurodegenerative pathologies and their combinations are more frequent than commonly believed in the elderly brain: a community-based autopsy series. Acta Neuropathol. 2013;126(3):365-84. https://doi.org/10.1007/s00401-013-1157-y.
34. Wirth M, Madison CM, Rabinovici GD, Oh H, Landau SM. Jagust WJ Alzheimer's disease neurodegenerative biomarkers are associated with decreased cognitive function but not beta-amyloid in cognitively normal older individuals. J Neurosci. 2013;33(13):5553-63. https://doi.org/10. 1523/JNEUROSCI.4409-12.2013.

35. Khoonsari PE, Shevchenko G, Herman S, Remnestal J, Giedraitis V, Brundin $R$, et al. Improved differential diagnosis of Alzheimer's disease by integrating ELISA and mass spectrometry-based cerebrospinal fluid biomarkers. J Alzheimers Dis. 2019;67(2):639-51. https://doi.org/10.3233/JAD-180855.

36. Vos SJB. Duara R The prognostic value of ATN Alzheimer biomarker profiles in cognitively normal individuals. Neurology. 2019;92(14):643-4. https://doi.org/10.1212/WNL.00000000000007223.

37. Haense C, Buerger K, Kalbe E, Drzezga A, Teipel SJ, Markiewicz P, et al. CSF total and phosphorylated tau protein, regional glucose metabolism and dementia severity in Alzheimer's disease. Eur J Neurol. 2008;15(11):115562. https://doi.org/10.1111/j.1468-1331.2008.02274.x.

38. Kim JY, Park JH, Lee JJ, Huh Y, Lee SB, Han SK, et al. Standardization of the Korean version of the geriatric depression scale: reliability, validity, and factor structure. Psychiatry Investig. 2008;5(4):232-8. https://doi.org/10. 4306/pi.2008.5.4.232.

39. Kang Y, Na DL. Hahn S A validity study on the Korean Mini-Mental State Examination (K-MMSE) in dementia patients. J Korean Neurol Assoc. 1997;15(2):300-8.

40. Wechsler D. Wechsler Memory Scale IV (WMS-IV). New York: Psychological Corporation; 2009.

41. Choi KY, Lee JJ, Gunasekaran TI, Kang S, Lee W, Jeong J, et al. APOE promoter polymorphism-219T/G is an effect modifier of the influence of APOE epsilon4 on Alzheimer's disease risk in a multiracial sample. J Clin Med. 2019;8(8). https://doi.org/10.3390/jcm8081236.

42. Fischl B. Dale AM Measuring the thickness of the human cerebral cortex from magnetic resonance images. Proc Natl Acad Sci U S A. 2000;97(20):11050-5. https://doi.org/10.1073/pnas.200033797.

43. Fischl B, Sereno MI, Tootell RB, Dale AM. High-resolution intersubject averaging and a coordinate system for the cortical surface. Hum Brain Mapp 1999;8(4): 272-284. doi: https://doi.org/10.1002/(sici) 1097-0193(1999)8:4

44. Fischl B, Salat DH, Busa E, Albert M, Dieterich M, Haselgrove C, et al. Whole brain segmentation: automated labeling of neuroanatomical structures in the human brain. Neuron. 2002;33(3):341-55. https://doi.org/10.1016/ s0896-6273(02)00569-x.

45. Klein A, Tourville J. 101 labeled brain images and a consistent human cortical labeling protocol. Front Neurosci. 2012;6171. https://doi.org/10. 3389/fnins.2012.00171.

46. Hwang J, Jeong JH, Yoon SJ, Park KW, Kim EJ, Yoon B, et al. Clinical and biomarker characteristics according to clinical spectrum of Alzheimer's disease (AD) in the validation cohort of Korean Brain Aging Study for the early diagnosis and prediction of AD. J Clin Med. 2019;8(3). https://doi. org/10.3390/jcm8030341.

47. Han JY, Seo EH, Yi D, Sohn BK, Choe YM, Byun MS, et al. A normative study of total scores of the CERAD neuropsychological assessment battery in an educationally diverse elderly population. Int Psychogeriatr. 2014;26(11):1897-904. https://doi.org/10.1017/S1041610214001379.

48. Hayes AF. Introduction to mediation, moderation, and conditional process analysis: a regression-based approach. New York: The Guilfor Press; 2013.

49. Hosmer DW Jr. Lemeshow S. Sturdivant RX. Applied logistic regression. New Jersey: Wiley; 2013.

50. Nie J, Duan L, Yan Z. Niu Q Tau hyperphosphorylation is associated with spatial learning and memory after exposure to benzo[a]pyrene in SD rats. Neurotox Res. 2013;24(4):461-71. https://doi.org/10.1007/ s12640-013-9387-2.

51. Braak H, Braak E. Neuropathological stageing of Alzheimer-related changes. Acta Neuropathol. 1991;82(4):239-59. https://doi.org/10.1007/ BF00308809.

52. Dowling NM, Johnson SC, Gleason CE, Jagust WJ. Alzheimer's Disease Neuroimaging I The mediational effects of FDG hypometabolism on the association between cerebrospinal fluid biomarkers and neurocognitive function. Neuroimage. 2015:105357-68. https://doi.org/10.1016/j.neuro image.2014.10.050.

53. Saint-Aubert L, Almkvist O, Chiotis K, Almeida R, Wall A, Nordberg A. Regional tau deposition measured by [(18)F]THK5317 positron emission tomography is associated to cognition via glucose metabolism in 
Alzheimer's disease. Alzheimers Res Ther. 2016;8(1):38. https://doi.org/10. 1186/s13195-016-0204-z.

54. Berry DTR, Allen RS. Schmitt FA Rey-Osterrieth complex figure: psychometric characteristics in a geriatric sample. Clin Neuropsychol. 1991;5(2):143-53. https://doi.org/10.1080/13854049108403298.

55. Youn YC, Pyun JM, Ryu N, Baek MJ, Jang JW, Park YH, et al. Use of the clock drawing test and the Rey-Osterrieth complex figure test-copy with convolutional neural networks to predict cognitive impairment. Alzheimers Res Ther. 2021:13(1):85. https://doi.org/10.1186/s13195-021-00821-8.

56. Gallagher C. Burke T Age, gender and IQ effects on the Rey-Osterrieth complex figure test. Br J Clin Psychol. 2007;46(Pt 1):35-45. https://doi.org/ 10.1348/014466506x106047

57. Kulason S, Xu E, Tward DJ, Bakker A, Albert M, Younes L, et al. Entorhinal and transentorhinal atrophy in preclinical Alzheimer's disease. Front Neurosci. 2020;14804. https://doi.org/10.3389/fnins.2020.00804.

58. Lopez ME, Bruna R, Aurtenetxe S, Pineda-Pardo JA, Marcos A, Arrazola $J$, et al. Alpha-band hypersynchronization in progressive mild cognitive impairment: a magnetoencephalography study. J Neurosci. 2014;34(44):14551-9. https://doi.org/10.1523/JNEUROSCI.0964-14.2014.

59. Ritchie C, Smailagic N, Noel-Storr AH, Ukoumunne O, Ladds EC, Martin S. CSF tau and the CSF tau/ABeta ratio for the diagnosis of Alzheimer's disease dementia and other dementias in people with mild cognitive impairment (MCI). Cochrane Database Syst Rev. 2017;3:CD010803. https://doi.org/10.1002/14651858.CD010803.pub2.

60. Palmqvist S, Mattsson N, Hansson O. Alzheimer's Disease Neuroimaging I Cerebrospinal fluid analysis detects cerebral amyloid-beta accumulation earlier than positron emission tomography. Brain. 2016;139(Pt 4):1226-36. https://doi.org/10.1093/brain/aww015.

61. Bateman RJ, Xiong C, Benzinger TL, Fagan AM, Goate A, Fox NC, et al. Clinical and biomarker changes in dominantly inherited Alzheimer's disease. N Engl J Med. 2012;367(9):795-804. https://doi.org/10.1056/NEJMo a1202753.

62. Aschenbrenner AJ, Gordon BA, Benzinger TLS, Morris JC. Hassenstab JJ Influence of tau PET, amyloid PET, and hippocampal volume on cognition in Alzheimer disease. Neurology. 2018;91(9):e859-66. https://doi.org/10. 1212/WNL.00000000000006075.

\section{Publisher's Note}

Springer Nature remains neutral with regard to jurisdictional claims in published maps and institutional affiliations.
Ready to submit your research? Choose BMC and benefit from:

- fast, convenient online submission

- thorough peer review by experienced researchers in your field

- rapid publication on acceptance

- support for research data, including large and complex data types

- gold Open Access which fosters wider collaboration and increased citations

- maximum visibility for your research: over 100M website views per year

At BMC, research is always in progress.

Learn more biomedcentral.com/submissions 